\title{
A method for examining short-term retention of haptic cues in monkeys
}

\author{
TERRANCE L. STEELE and RICHARD H. BAUER \\ Middle Tennessee State University, Murfreesboro, Tennessee
}

\begin{abstract}
Tasks used to examine short-term memory (STM) in animals have almost exclusively required retention of visual cues. To determine if haptic information can be retained, three rhesus monkeys were trained to perform, using only haptic cues, a simultaneous (SMS) and a delayed matching-to-sample (DMS) task. On each trial, the monkeys felt and responded to a sample stimulus on a centrally located manipulandum. They were then presented two comparison stimuli located on both sides of the central manipulandum. A response matching the comparison stimulus with the sample stimulus was reinforced. In SMS a mean of 2,725 trials was required to reach a criterion of $90 \%$ correct. As in DMS performance for visual cues, in haptic DMS the monkeys were capable of above-chance responding at retention intervals of greater than $1 \mathrm{~min}$. This haptic DMS task should be useful for testing STM models, for examining the physiological basis of STM, and for examining drug effects.
\end{abstract}

Delayed matching to sample (DMS) is currently the most widely used task to examine short-term memory (STM) in animals. The typical DMS task involves presentation of a visual stimulus (sample), followed by a response to and the subsequent removal of that sample. At the end of a brief retention interval, the same stimulus reappears in a different position, accompanied by one or more comparison stimuli of the same modality. A response to the comparison stimulus that is identical to the sample is reinforced.

The DMS task has become widely used in animal STM studies because, unlike other tasks requiring shortterm retention (delayed response, delayed alternation, and some mazes), the animal is unlikely to use body orientation as a cue for correct responding (French, 1959; Miles, 1957). In addition, in DMS the relevant cues are visual, whereas in the other tasks the relevant cues cannot be specified. There are, in fact, very few animal STM tasks in which the relevant cues are not exclusively visual. Monkeys have been trained to match auditory and visual cues when there is a delay between the two cues (Dewson \& Burlingame, 1975). Monkeys can respond differentially to tone pairs of the same or different frequencies when there is a delay of $1-5 \mathrm{sec}$ between the pairs; but performance changes across delays were not reported (Stepien \& Cordeau, 1960). However, attempts to train monkeys in DMS with only auditory cues have been unsuccessful (D'Amato, 1973), and training animals in DMS with only haptic cues has not been attempted. To our knowledge, the only animal

We thank Loren Evey for technical assistance and Charles Casper, Mortazu Irannezhud, and Jack Dodge for assistance in training the monkeys. Our mailing address is: Department of Psychology, Middle Tennessee State University, Murfreesboro, Tennessee 37132. task that has not involved visual cues was a DMS task in which a dolphin was trained to match auditory cues (Herman \& Gordon, 1974).

As indicated above, researchers interested in shortterm retention in animals have relied almost exclusively on tasks requiring retention of visual cues. Since monkeys can be trained to perform haptic and visual discriminations at about the same rate (Ettlinger, Morton, \& Moffett, 1966; Jarrard \& Moise, 1971), it appears likely that monkeys can be trained in a short-term retention task with only haptic cues. In the present experiment, we describe a method by which monkeys were trained to perform a DMS task with only haptic cues.

\section{METHOD}

\section{Subjects}

Three experimentally naive, male rhesus monkeys (Macaco mulatta) weighing 7-10 kg served as subjects. Throughout the experiment, the animals were housed individually and had free access to Purina monkey chow, which was supplemented with fruit. On training days, all fluids were obtained as reinforcement and no fluids were available in the home cage. Daily fluid consumption during this perjod was approximately $60 \%$ of ad-lib consumption.

\section{Apparatus}

Training was conducted in a sound-attenuated room adjacent to the colony room. The monkeys were placed in a primate chair with a stainless steel tube positioned in front of the mouth for delivery of a $1.5-\mathrm{ml}$ grape juice reinforcement. The right hand was loosely chained, so that all responses were made with the left hand. Training was conducted in a modified Lehigh Valley Electronics primate test chamber $(60 \times 60 \times 73 \mathrm{~cm})$. The chair was positioned in the chamber in such a way that the monkeys could easily reach through a triangular hole $(15.0 \mathrm{~cm}$ wide, $8.5 \mathrm{~cm}$ from base to peak, and $26.5 \mathrm{~cm}$ above the chamber floor) in the front wall (Figure 1). Three manipulanda $(10.5 \mathrm{~cm}$ long, $0.5 \mathrm{~cm}$ in diameter, and $5.0 \mathrm{~cm}$ apart), were attached by hinges to the outside wall immediately in front of the hole. The base of each manipulandum was connected to a microswitch. 


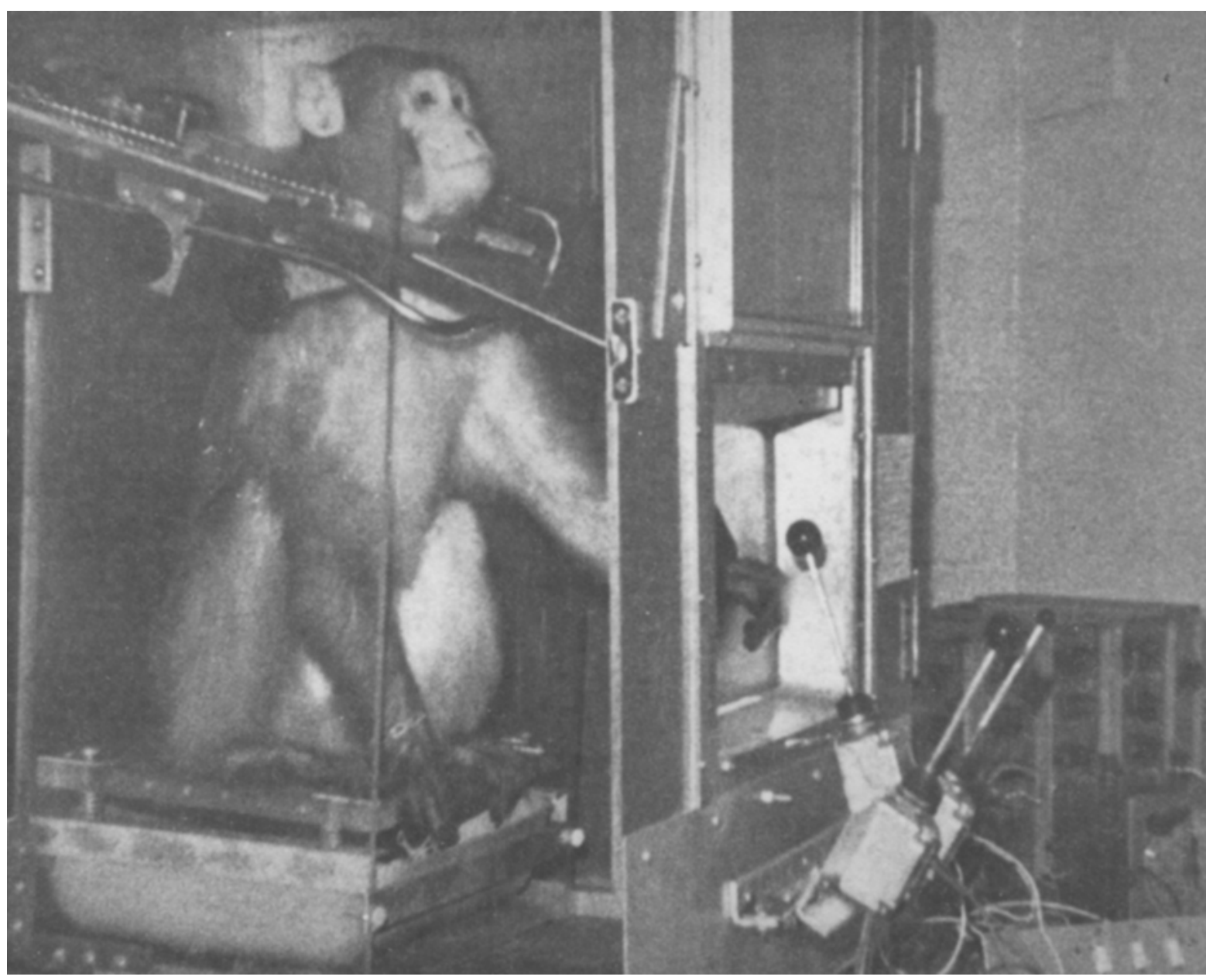

Figure 1. A monkey and primate chair positioned within the test chamber. The side wall has been removed for viewing. Haptic stimuli mounted on the manipulanda are shown on the right. The sample stimulus has been moved forward and the comparison stimuli are retracted.

Closure of a microswitch required a 10-deg movement of the manipulanda in any direction and produced an audible click. The tips of the manipulanda formed an isosceles triangle, with the center manipulandum being $2.0 \mathrm{~cm}$ above the outer manipulanda. Small objects (1.5-2.5 cm across), such as spheres, cubes, knobs, hooks, and pieces of Plexiglass cut into various shapes, served as the haptic stimuli during training. These objects were permanently attached to aluminum tubing $(9.0 \mathrm{~cm}$ long) that could be easily slipped on and off the manipulanda. A metal plate, extending from the neck to a position above the hole in the chamber wall, prevented the monkeys from seeing the stimuli. The experimenter manually presented the manipulanda so that the stimuli were immediately in front of the hole (Figure 1). Retention intervals and intertrial intervals were measured by Hunter timers, which were started by responses to the manipulanda. After initial training, a shaded $75-\mathrm{W}$ bulb, located outside the test chamber, served as the only source of illumination. Although illumination outside the chamber was adequate for data recording, illumination within the test chamber was minimal. A fan provided ventilation and masked extraneous noise.

\section{Training Procedure}

The monkeys were trained according to a procedure analogous to that described in DMS with visual cues (Bauer \& Fuster, 1976; Moise \& Jarrard, 1969). The monkeys were first shaped, by successive approximations, to pull the manipulanda; no objects were on the manipulanda during this phase. Responses to the outer manipulanda were alternately reinforced and not reinforced until very few responses were made to the nonreinforced manipulandum. During the first few shaping sessions, the neck plate was removed and the hole was widened to $24 \mathrm{x}$ $29 \mathrm{~cm}$ so that the monkeys had a clear view of the manipulanda. During the following sessions, the smaller triangular hole was restored and the neck plate was replaced in its original position so that the stimuli could not be seen. The monkeys were subsequently trained to discriminate between two objects placed on the outer manipulanda (e.g., a knob and wing nut), and this was followed by 10 to 15 discrimination reversals. A criterion of 18 correct choices out of 20 consecutive trials was required.

The monkeys were next trained on a simultaneous matchingto-sample (SMS) task using the same two objects that had been used during discrimination and reversal training. An object on the center manipulandum (the sample stimulus) was presented to the monkeys. Presentation of a sample was signaled by lightly tapping the center manipulandum on the outside wall of the test chamber. The monkeys were allowed to feel the sample object and to pull it with enough force to close the microswitch; this defined a response. After the monkeys had responded to the sample stimulus, the two objects on the outer manipulanda (the 
comparison stimuli) were presented as rapidly as possible (within $.5 \mathrm{sec}$ ). Although actual simultaneous haptic perception of all three stimuli was not possible, this procedure is called SMS, for purposes of comparison with other studies. One of the comparison objects was always identical to the sample stimulus, and a response to this object was reinforced with grape juice. The trial terminated when a response was made to either comparison stimulus or if no response was made within $5 \mathrm{sec}$ after presentation of the comparison stimuli. The monkeys were allowed to touch both stimuli throughout the 5 -sec time limit. In the first phase of SMS training, across trials, the same sample was presented and the positions of the comparison stimuli were random until approximately $90 \%$ of the monkeys' responses were correct. Then, the same procedure was followed with the other sample stimulus. This was followed by several reversals of the sample stimuli, and gradually, across training sessions, the two sample stimuli and the positions of the comparison stimuli were presented randomly. The intertrial interval (ITI) was $30 \mathrm{sec}$. The monkeys were required to reach a criterion of 90 correct choices out of 100 consecutive trials, followed by approximately 100 overtraining trials. A mean of 250 trials per session was given.

To determine whether training would transfer across problems, the first monkey (Chompers) was subsequently trained in seven more SMS problems, with two different objects per problem. The other monkeys (Ollie and Harry) received two and three additional problems, respectively.

All the monkeys were then given 13 to 27 daily sessions in which the stimuli were cubes and spheres with sides or diameters of 12,24 , and $36 \mathrm{~mm}$. These objects were coated with polyester casting resin (Chemco) to ensure that each had a uniform surface. The monkeys were first trained to make what appeared to be easy matches with spheres, such as large versus small. This was followed by either pairing the small with the medium sphere and the medium with the large sphere (for Chompers) or by presenting combinations of all three spheres (for Ollie and Harry). The same procedure was then followed with cubes. In the last several sessions, random presentation of all possible combinations of any two stimuli was given.

In the final training phase, a retention interval of $.5,2,4$, $8,16,32$, or $64 \mathrm{sec}$ was imposed between the response to the sample and presentation of the comparison stimuli. The monkeys received 12 daily sessions of 180 trials each. Across the first 4 sessions, the retention intervals increased from .5 to $8 \mathrm{sec}$, with only one interval given per session. The $16-\mathrm{sec}$ interval was presented during the next 2 sessions, the 32 -sec interval during the next 3 sessions, and the 64-sec interval during the final 3 sessions. With the three longest delays, trials of shorter intervals were interspersed to ensure continued responding during the session. Every retention interval was presented 340 times over the 12 sessions, with the exception of the 64-sec retention interval, which was presented 120 times. During this training phase, the monkeys were allowed to touch the sample for a maximum of $1 \mathrm{sec}$.

\section{RESULTS}

One session was required to shape each animal to pull one of the manipulanda, and a mean of six sessions was required to pull each of the manipulanda equally often. A mean of 994 trials (four daily sessions) was necessary to reach a criterion of $90 \%$ correct responses in 20 trials on the first discrimination problem (Table 1). Training on the first reversal required a mean of 474 trials to criterion. Across the remaining reversals, all monkeys were able to reach criterion in less than 50 trials. A mean of 2,725 trials, or approximately 11 sessions, was required for the monkeys to reach a criterion of $90 \%$ correct responses in 100 trials on the first SMS problem. Thus, the total procedure necessary for shaping, discrimination training, reversals, and SMS required a mean of 30 daily sessions (Chompers $=18$; Ollie $=38$; Harry $=34$ ). Not including initial shaping, this comprised a mean of 5,854 trials (Chompers = 2,$106 ;$ Ollie $=9,353 ;$ Harry $=6,103$ ).

To determine if fewer trials to criterion would occur across different SMS problems, Chompers was given seven additional SMS problems. Ollie and Harry were given only two and three additional SMS problems, respectively, due to the extensive number of trials necessary to reach criterion on these problems. Different pairs of stimuli were used on each problem. No decrease in trials to criterion was observed across successive problems, except for Ollie, who reached criterion on the second two problems in 1,835 and 1,966 trials (compared with 6,332 trials on the first problem).

SMS training with spheres and cubes had to be adjusted according to the progress of the individual monkeys. The number of SMS trials given to each monkey is shown in Table 1. Chompers received pairings of small and large, small and medium, and medium and large spheres, respectively. The same order of pairings was given with cubes. Chompers reached the criterion of 90-out-of-100 correct matches in 162 trials when all

Table 1

Number of Trials to Criterion for Individual Subjects During Each Stage of Training

\begin{tabular}{|c|c|c|c|c|c|c|c|c|c|}
\hline & \multirow[b]{2}{*}{$\begin{array}{c}\text { Discrim- } \\
\text { ination }\end{array}$} & \multicolumn{3}{|c|}{ Reversals (R) } & \multicolumn{5}{|c|}{ Simultaneous Matching to Sample (SMS) } \\
\hline & & $\begin{array}{c}1 \mathrm{st} \\
\mathrm{R}\end{array}$ & $\begin{array}{c}\text { Range of } \\
\text { Remain- } \\
\text { ing } R\end{array}$ & $\begin{array}{l}\text { Total No. } \\
\text { Trials of } \\
\text { Remain- } \\
\text { ing } R\end{array}$ & $\begin{array}{l}\text { 1st SMS } \\
\text { Problem }\end{array}$ & $\begin{array}{c}\text { Range } \\
\text { (Additional } \\
\text { SMS } \\
\text { Problems) }\end{array}$ & $\begin{array}{c}\text { SMS } \\
\text { (Spheres } \dagger \text { ) }\end{array}$ & $\begin{array}{c}\text { SMS } \\
\text { (Cubes } \dagger \text { ) }\end{array}$ & $\begin{array}{c}\text { SMS } \\
\text { (All Com- } \\
\text { binations) }\end{array}$ \\
\hline $\begin{array}{l}\text { Chompers } \\
\text { Ollie } \\
\text { Harry }\end{array}$ & $\begin{array}{c}352 \\
1154^{*} \\
1476\end{array}$ & $\begin{array}{l}328 \\
541 \\
554\end{array}$ & $\begin{array}{l}227-20 \\
338-44 \\
449-30\end{array}$ & $\begin{array}{r}978 \\
1271 \\
2704\end{array}$ & $\begin{array}{r}435 \\
6332 \\
1408\end{array}$ & $\begin{array}{l}1853-299 * * \\
1966-1835 \\
4199-1674 * *\end{array}$ & $\begin{array}{r}1054 \\
431 \\
1964\end{array}$ & $\begin{array}{r}366 \\
1333 \\
2258\end{array}$ & $\begin{array}{l}162 \\
168 \\
1636 * *\end{array}$ \\
\hline Mean & 994 & 474 & $338-31$ & & 2725 & & 1150 & 1319 & \\
\hline
\end{tabular}

* Ollie responded at chance levels for 593 trials with a cylindrical knob and T-shaped bar but reached criterion in 561 trials with a knob and wingnut. $\quad * *$ Subject did not meet criterion on all problems. TCriterion levels were not established for these problems. 


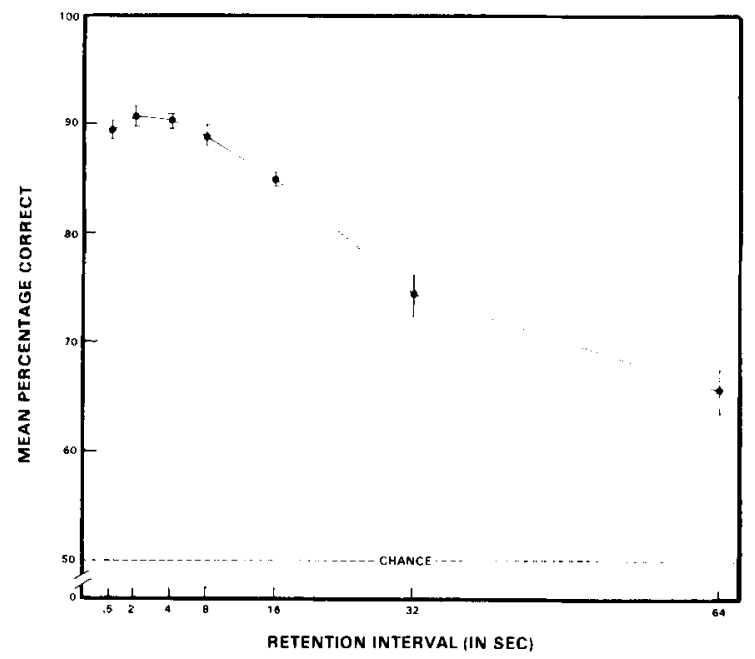

Figure 2. Mean percentage correct in DMS as a function of the retention interval. A vertical line at each data point indicates the standard error of the mean.

possible combinations of spheres and cubes were given. Ollie and Harry reached criterion with small and large spheres, but responded at near-chance levels when small and medium spheres and medium and large spheres were presented. However, matching errors by Ollie and Harry decreased when all three pairings of spheres were presented in a mixed order. A similar problem occurred with cubes, and the same correction was given. Ollie reached criterion in 168 trials with all combinations of spheres and cubes, whereas Harry was unable to reach criterion after 1,636 trials.

Retention-interval training covered 12 daily sessions. Retention intervals were presented sequentially across sessions from the shortest to the longest, and, for this reason, retention intervals and training sessions are confounded. Nevertheless, changes in percentage-correct responding across retention intervals are of interest, and therefore these data are presented (Figure 2). A 3 (monkeys) $\times 7$ (retention intervals) mixed analysis of variance on the percentage correct during the last 12 sessions showed that there was no significant difference among the monkeys, but that there was a significant decrease as a function of the retention interval $[F(6,12)$ $=28.25, \mathrm{p}<.001]$.

\section{DISCUSSION}

Several problems with the training procedures were encountered. Occasionally, monkeys did not respond to the stimulus objects, but instead pulled the manipulanda beneath the objects. To correct this problem, after the animals had been shaped to pull the manipulanda, the shape of the hole was adjusted so that the tips of the manipulanda were at the same level as the lower edge of the hole (see Figure 1). This made it more likely that the monkeys would touch the stimulus objects. Even with this modification, one monkey continued to pull the manipulanda below the objects. This problem was solved by placing the experimenter's hands loosely beneath the outer objects, thus ensuring that the monkey felt the objects. Occasional position and object biases were eliminated by a correction procedure or by firmly holding the base of the manipulandum on the favored side, so that the object could be felt but not pulled.

Since multiple responses to visual samples have been found to decrease DMS errors (Jarrard \& Moise, 1971), three or more responses to the sample stimulus were occasionally required to ensure that the monkey was feeling the sample. However, the requirement of multiple responses seemed to have little effect on improvement in the monkey's performance.

Some of the training procedures used on the first monkey (Chompers) appeared to be unnecessary (or the lengths of time spent on these procedures were considered to be longer than needed). These were therefore shortened when the second monkey (Ollie) was trained. This strategy may have been detrimental in SMS training with spheres and cubes. Because of Ollie's slow rate of acquisition, no procedures were shortened in training Harry.

The monkeys in the present experiment learned the first haptic discrimination in a number of trials roughly comparable to the number required in other haptic discriminations (Ettinger, Morton, \& Moffett, 1966) and auditory discriminations for frequency and intensity (Wegener, 1964). Visual discrimination of flickering lights was learned in approximately the same number of trials, but color and pattern discriminations were acquired more rapidly (Jarrard \& Moise, 1971). A criterion level of $90 \%$ correct responses was required in all of the above studies, but there were a number of differences in apparatus and procedure, in addition to the differences in cue modality. Given the differences among these studies, the results are surprisingly consistent across studies. The consistency of these findings suggests that monkeys learn haptic, auditory, and some visual discriminations at about the same rate.

In some instances, SMS acquisition with haptic cues is comparable to SMS acquisition with other modalities. As described in the procedure section, one of the first steps in SMS training involves presenting the same sample stimulus across trials until some criterion is reached and then the other sample until the same criterion is reached. A number of reversals are then given with these samples. Monkeys require fewer trials to reach criterion across successive reversal, and the decrease across reversals is comparable for haptic and visual cues (Bauer \& Fuster, Note 1). Using a comparable procedure with auditory cues, Thompson (1980) reported that only one of four monkeys showed any decrease in trials to criterion across reversals and that none of the monkeys learned SMS with auditory cues. Thus, monkeys have greater difficulty in acquiring SMS with auditory cues than with haptic and visual cues. In support of this suggestion, 
D'Amato (1973) and his co-workers were unable to train monkeys to perform SMS with auditory cues, although they have had extensive experience in training monkeys in SMS with visual cues.

Although there are no studies that have reported the successful training of monkeys in SMS and DMS with only auditory cues, Dewson and Burlingame (1975) trained monkeys to perform a conditional matching task in which monkeys responded to a red panel after hearing a $1,000 \cdot \mathrm{Hz}$ tone and to a green panel after hearing a burst of white noise. The first few animals required approximately 49,500 trials to criterion, but after Dewson and Burlingame had had several years of experience in training animals in this task, about 12,000 trials were necessary (Dewson, Note 2). The greater number of trials required in Dewson's task may be due to the conditional matching procedure (D'Amato \& Worsham, 1974), to the cross-modal nature of the task (Davenport, Rogers, \& Russell, 1973), or to the auditory cues' being learned slowly. Although monkeys may acquire haptic, visual, and auditory SMS tasks at a faster rate when better training procedures are used, it is important to note that with currently available techniques, the amount of training necessary for monkeys to learn discriminations and SMS with haptic and visual cues is less than that necessary for auditory cues. Comparable acquisition rates for visual and haptic cues occur in spite of the fact that visual cues are presented simultaneously, whereas haptic and auditory cues are presented sequentially and must be retained until a response has been made.

The degree to which monkeys transfer to new sample stimuli in SMS may be lower with haptic and auditory cues than with visual cues. With visual cues, monkeys generalize to new samples (D'Amato, 1973; Worsham, 1975). In the experiment reported here, only one monkey (Ollie) reached criterion in fewer trials on the second and third SMS problems than on the first. However, this decrease may have been due not to transfer across problems but to the greater difficulty of the first SMS problem. Thompson (Note 3) reported that, with auditory cues, one monkey generalized to new sample stimuli; in another experiment, three other monkeys could not learn the first SMS problem (Thompson, 1980).

The change in percentage correct across retention intervals in the present study is very similar to the change in DMS tasks using visual cues (Bauer \& Fuster, 1976; Jarrard \& Moise, 1971, Figure 9). Whenever possible, the training procedures used here were the same as those used in visual DMS training (Bauer \& Fuster, 1976; Jarrard \& Moise, 1971); similarity in training procedures may have contributed to comparable performance in haptic and visual DMS. The similar decrease in percentage correct as a function of the retention interval in haptic and visual DMS indicates that monkeys can retain haptic and visual cues to a comparable degree.
In a conditional matching task that required a response to a red panel after a $1,000-\mathrm{Hz}$ tone was heard and a response to a green panel after a burst of white noise was heard, there was a much greater decrease in percentage correct as a function of the retention interval (Dewson \& Burlingame, 1975) than is evident in DMS with haptic and visual cues. The greater decrease across retention intervals in the auditory-visual matching task may be due to the conditional matching procedure required in this task, the cross-modal nature of the task, or the greater difficulty of retaining auditory information than of retaining visual or haptic information.

Establishment of an animal haptic STM task provides a basis for making comparisons with STM processes in other modalities. For example, procedures that affect the retention of visual cues, such as presentation of visual stimuli during the retention interval, can be tested with haptic stimuli to ascertain whether the effect is modality specific. In general, the haptic STM task can be used to verify and broaden many of the findings and theories about animals' STM, which theories have been almost exclusively based on visual tasks. Furthermore, the haptic DMS task can be a useful tool for investigating the physiological bases of STM and for investigating drug effects.

\section{REFERENCE NOTES}

1. Bauer, R. H., \& Fuster, J. M. Unpublished observations, 1976.

2. Dewson, J. H., III. Toward an animal model of auditory cognitive function. In The neurological bases of language disorders in children: Methods and directions for research. Symposium presented at the meeting of the National Institute of Neurological and Clinical Disease and Stroke, Bethesda, Maryland, 1978.

3. Thompson, R. K. R. Nonconceptual auditory matching by a rhesus monkey reflects biological constraints on cognitive processes? Paper presented at the Northeast Regional Meeting of the Animal Behavior Society, Kingston, Ontario, Canada, 1981.

\section{REFERENCES}

Bauer, R. H., \& Fuster, J. M. Delayed-matching and delayedresponse deficit from cooling dorsolateral prefrontal cortex in monkeys. Journal of Comparative and Physiological Psychology, 1976, 90, 293-302.

D'Amato, M. R. Delayed matching and short-term memory in monkeys. In G. H. Bower (Ed.), The psychology of learning and motivation: Advances in research and theory (Vol. 7). New York: Academic Press, 1973.

D'Aмато, M. R., \& Wonshaм, R. W. Retrieval cues and shortterm memory in capuchin monkeys. Journal of Comparative and Physiological Psychology, 1974, 86, 274-282.

Davenpokt, R. K., Rogers, C. M., \& Russell, I. S. Cross modal perception in apes. Neuropsychologia, 1973, 11, $21-28$.

Dewson, J. H., III, \& Burlingame, A. C. Auditory discrimination and recall in monkeys. Science, 1975, 187, 267-268.

Ettlingen, G., Morton, H. B., \& Moffett, A. Tactile discrimination performance in the monkey: The effect of bilateral posterior parietal and lateral frontal ablations, and of callosal section. Cortex, 1966, 2, 5-29.

French, G. M. Performance of squirrel monkeys on variants of delayed response. Journal of Comparative and Physiological Psychology, 1959, 52, 741-745.

Herman, L. M., \& Gordon, J. A. Auditory delayed matching in 
the bottlenose dolphin. Journal of the Experimental Analysis of Behavior, 1974, 21, 19-26.

JARRARD, L. E., \& Moise, S. L. Short-term memory in the monkey. In L. E. Jarrard (Ed.), Cognitive processes of nonhuman primates. New York: Academic Press, 1971.

Miles, R. C. Delayed-response learning in the marmoset and the macaque. Journal of Comparative and Physiological Psychology, 1957, 50, 352-355.

Moise, S. L., \& JARRaRd, L. E. A computer-controlled system for training and testing primates. Behavior Research Methods \& Instrumentation, 1969, 1, 234-236.

Stepien, L. S., \& Corde aU, J. P. Memory in monkeys for compound stimuli. American Journal of Psychology, 1960, 73, 388-395.
Thompson, R. K. R. Auditory cued reversal and matching-tosample learning by rhesus monkeys. Antropologia Contemporanea, 1980, 3, 284.

Wegener, J. G. Auditory discrimination behavior of normal monkeys. Journal of Auditory Research, 1964, 4, 81-106.

Worsham, R. W. Temporal discrimination factors in the delayed matching-to-sample task in monkeys. Animal Learning \& Behavior, 1975, 3, 93-97.

(Manuscript received July 13, 1982;

revision accepted for publication February 1, 1983.) 